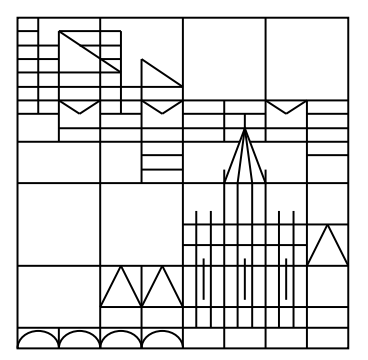

\title{
Convergence of Nonlinear Schrödinger-Poisson Systems to the Compressible Euler equations
}

\author{
Ansgar Jüngel \\ Shu Wang
}

Konstanzer Schriften in Mathematik und Informatik

Nr. 172, April 2002

ISSN 1430-3558

Konstanzer Online-Publikations-System (KOPS)

URL: http://www.ub.uni-konstanz.de/kops/volltexte/2007/2195/

URN: http://nbn-resolving.de/urn:nbn:de:bsz:352-opus-21953

(c) Fachbereich Mathematik und Statistik

(c) Fachbereich Informatik und Informationswissenschaft

Universität Konstanz

Fach D 188, 78457 Konstanz, Germany

Email: preprints@informatik.uni-konstanz.de

WWW: http://www.fmi.uni-konstanz.de/Schriften 


\title{
Convergence of Nonlinear Schrödinger-Poisson Systems to the Compressible Euler equations
}

\author{
Ansgar Jüngel ${ }^{1}$ \\ Fachbereich Mathematik und Statistik, Universität Konstanz, \\ Fach D193, 78457 Konstanz, Germany, \\ e-mail: juengel@fmi.uni-konstanz.de \\ Shu Wang ${ }^{2}$ \\ Institut für Mathematik, Universität Wien, \\ Boltzmanngasse 9, 1090 Wien, Austria \\ and \\ Academy of Mathematics and System Sciences, CAS, Beijing 100080, \\ P. R. China, e-mail: shu.wang@univie.ac.at
}

\begin{abstract}
The combined semi-classical and quasineutral limit in the bipolar defocusing nonlinear Schrödinger-Poisson system in the whole space is proven. The electron and current densities, defined by the solution of the Schrödinger-Poisson system, converge to the solution of the compressible Euler equation with nonlinear pressure. The corresponding Wigner function of the Schrödinger-Poisson system converges to a solution of a nonlinear Vlasov equation. The proof of these results is based on estimates of a modulated energy functional and on the Wigner measure method.
\end{abstract}

2000 Mathematics Subject Classification: 35Q40, 35Q53, 35Q55, $76 \mathrm{Y} 07$.

Keywords: Bipolar defocusing nonlinear Schrödinger-Poisson systems, compressible Euler equations, semi-classical limit, quasineutral limit, Wigner measure.

\footnotetext{
${ }^{1}$ Supported by the Deutsche Forschungsgemeinschaft, grants JU 359/3 (Gerhard-Hess Program) and JU 359/5 (Priority Program "Multiscale Problems"), the TMR Project "Asymptotic methods in kinetic theory" (grant ERB-FMBX-CT97-0157) and the AFF Project of the University of Konstanz.

${ }^{2}$ Supported by the National Youth Natural Science Fundation (grant 10001034) of China and the Wittgenstein-Award of Peter A. Markowich's FTW (Austria). I would like to thank Professors Y. Brenier, N. Mauser and P. A. Markowich for many useful discussions. I also wish to express my gratitude to Professor P. A. Markowich. Without his help and friendly advice, this work would not have been possible.
} 


\section{Introduction}

The main objective of this paper is to study the combined semiclassical and quasineutral limit of the bipolar defocusing nonlinear Schrödinger-Poisson in $\mathbb{R}^{d}(d \geq 1)$ :

$$
\begin{aligned}
i \varepsilon \partial_{t} \Psi_{j} & =-\frac{\varepsilon^{2}}{2} \Delta \Psi_{j}+\left(q_{j} V+h\left(\left|\Psi_{j}\right|^{2}\right)\right) \Psi_{j}, \quad x \in \mathbb{R}^{d}, t>0, \\
-\lambda^{2} \Delta V & =\left|\Psi_{1}\right|^{2}-\left|\Psi_{2}\right|^{2}, \quad x \in \mathbb{R}^{d}, t>0, \\
\Psi_{j}(0, \cdot) & =\Psi_{j I} \quad \text { in } \mathbb{R}^{d}, j=1,2 .
\end{aligned}
$$

The variables are the wave function $\Psi_{j}=\Psi_{j}(t, x), j=1,2$, and the electrostatic potential $V=V(t, x)$. The nonlinear self-interacting potential $h(s)$ is assumed to be given by

$$
h(s)=a^{2} s^{\gamma} \quad \text { for } s \geq 0, \text { for some } a>0, \gamma \geq 1 .
$$

The charges of the particles described by the wave functions $\Psi_{j}$ are defined by $q_{1}=1$ and $q_{2}=-1$, respectively. The scaled Planck constant $\varepsilon$ and the scaled Debye length $\lambda$ are given by

$$
\varepsilon^{2}=\frac{\hbar^{2}}{2 m k_{B} T L^{2}}, \quad \lambda^{2}=\frac{\varepsilon_{s} k_{B} T}{N q^{2} L^{2}},
$$

where $\hbar$ denotes the reduced Planck constant, $m$ the effective mass of the particles, $k_{B}$ the Boltzmann constant, $T$ the temperature constant, $\varepsilon_{s}$ the permittivity of the medium in which the particles are moving, $q$ the elementary charge, $N$ a characteristic density of the particles, and $L$ a characteristic length of the particle configuration. It is assumed that the effective mass and the characteristic density are the same for both particle species (otherwise additional factors have to be introduced). For instance, the system (1)-(3) describes the evolution of negatively and positively charged particles in a quantum plasma or in a semiconductor crystal with characteristic length $L$.

For this scaling of the equations we have assumed that the electrostatic potential is of the order of $k_{B} T / q$ and that the time is of the order of $\tau=2 m L^{2} / \hbar$. This means that we suppose that the kinetic energy of the particles needed to cross a domain of length $L$ in time $\tau$ is of the same order as the energy of a wave with frequency $1 / \tau$.

We are interested in the situation where the characteristic length $L$ is "large" (compared to the de Broglie length $\hbar / \sqrt{2 m k_{B} T}$ and to the Debye length $\left.\sqrt{\varepsilon_{s} k_{B} T / N q^{2}}\right)$. This means that both parameters $\varepsilon$ and $\lambda$ are "small" compared to one. In this paper we study the asymptotic limit $\varepsilon \rightarrow 0$ and $\lambda \rightarrow 0$. The limit $\varepsilon \rightarrow 0$ refers to as the semiclassical limit, the limit $\lambda \rightarrow 0$ as the quasineutral limit.

In order to formulate the limit equations corresponding to the system (1)-(2) for $\varepsilon=\lambda=0$, we use the fluiddynamical formulation of (1). Introduce the particle densities $n_{j}=\left|\Psi_{j}\right|^{2}$ and the current densities $J_{j}=\varepsilon \operatorname{Im}\left(\bar{\Psi}_{j} \nabla \Psi_{j}\right)$, and write the wave function as

$$
\Psi_{j}(x)=\sqrt{n_{j}(x)} \exp \left(i S_{j}(x) / \varepsilon\right), \quad j=1,2,
$$


where $S_{j}$ is the phase of $\Psi_{j}$ which is assumed to exist everywhere. Substituting this expression in (1) and separating real and imaginary parts, gives the irrotational flow equations $[6,16]$

$$
\begin{aligned}
\partial_{t} n_{j}+\operatorname{div}\left(n_{j} \nabla S_{j}\right) & =0, \\
\partial_{t} S_{j}+\frac{1}{2}\left|\nabla S_{j}\right|^{2}+q_{j} V+h\left(n_{j}\right)-\frac{\varepsilon^{2}}{2} \frac{\Delta \sqrt{n_{j}}}{\sqrt{n_{j}}} & =0,
\end{aligned}
$$

where $j=1,2$. Taking the gradient of (6), using the identity $J_{j}=n_{j} \nabla S_{j}$ and (5), we obtain the quantum hydrodynamic equations

$$
\begin{aligned}
\partial_{t} n_{j}+\operatorname{div} J_{j} & =0, \\
\partial_{t} J_{j}+\operatorname{div}\left(\frac{J_{j} \otimes J_{j}}{n_{j}}\right)+q_{j} n_{j} \nabla V+n_{j} \nabla h\left(n_{j}\right)-\frac{\varepsilon^{2}}{2} n_{j} \nabla\left(\frac{\Delta \sqrt{n_{j}}}{\sqrt{n_{j}}}\right) & =0, \\
-\lambda \Delta V & =n_{1}-n_{2},
\end{aligned}
$$

where the symbol $\otimes$ denotes the tensor product of vectors and $j=1,2$. The equations (7)-(8) are the fluiddynamical formulation of the nonlinear Schrödinger equation and are known as the Madelung equations $[16,17]$.

If we assume that the initial wave functions can be written as

$$
\Psi_{j I}(x)=\sqrt{n_{j I}(x)} \exp \left(i S_{j I}(x) / \varepsilon\right),
$$

the initial conditions to (7)-(8) are

$$
n_{j}(0, \cdot)=n_{j I}, \quad J_{j}(0, \cdot)=n_{j I} \nabla S_{j I} \quad \text { in } \mathbb{R}^{d}, j=1,2 .
$$

Furthermore, we suppose that the initial densities and the initial velocities are equal (in order to avoid initial layers):

$$
n_{0}:=n_{1 I}=n_{2 I}, \quad u_{0}:=\nabla S_{1 I}=\nabla S_{2 I} \quad \text { in } \mathbb{R}^{d} .
$$

The second assumption is not needed for our rigorous result, but it simplifies the following considerations.

Now, we set formally $\varepsilon=\lambda=0$ in (7)-(8). Then $n=n_{1}=n_{2}$ and (7)-(8) become

$$
\begin{aligned}
\partial_{t} n+\operatorname{div} J_{j} & =0, \\
\partial_{t} J_{j}+\operatorname{div}\left(\frac{J_{j} \otimes J_{j}}{n}\right)+q_{j} n \nabla V+n \nabla h(n) & =0 .
\end{aligned}
$$

Since the velocities $\nabla S_{1}$ and $\nabla S_{2}$ are equal initially, we get $J(t)=J_{1}(t)=J_{2}(t)$ for all $t>0$. We can eliminate the field $\nabla V$ by adding both equations (12) for $j=1,2$, using $q_{1}=-q_{2}=1$ and setting $u=\nabla S_{1}=\nabla S_{2}$ :

$$
\begin{aligned}
\partial_{t} n+\operatorname{div}(n u) & =0, \\
\partial_{t} u+u \cdot \nabla u+\nabla h(n) & =0, \quad x \in \mathbb{R}^{d}, t>0 .
\end{aligned}
$$


These are the classical Euler equations for compressible fluids (see [21]), complemented by the initial conditions $n(0, \cdot)=n_{0}$ and $u(0, \cdot)=u_{0}$ in $\mathbb{R}^{d}$.

The limit $\varepsilon \rightarrow 0, \lambda \rightarrow 0$ cannot be made directly in (7)-(9), since the phase $S_{j}$ or the quotients $1 / \sqrt{n_{j}}$ and $1 / n_{j}$ may be undefined. Therefore, we use another formulation of the Schrödinger-Poisson system (1)-(2), namely the Wigner equation. For this, we introduce the Wigner transform $[18,22]$

$$
w_{j}(t, x, v)=\frac{1}{(2 \pi)^{d}} \int_{\mathbb{R}^{d}} \bar{\Psi}_{j}\left(t, x+\frac{\eta}{2}\right) \Psi_{j}\left(t, x-\frac{\eta}{2}\right) e^{i \eta \cdot v} d \eta
$$

A simple calculation shows that $w_{j}$ satisfies the so-called Wigner equation

$$
\begin{aligned}
\partial_{t} w_{j}+v \cdot \nabla w_{j}+\Theta\left[q_{j} V+h\left(n_{j}\right)\right] w_{j} & =0, \quad x, v \in \mathbb{R}^{d}, t>0, \\
w_{j}(0, x, v) & =w_{j I}(x, v), \quad x, v \in \mathbb{R}^{d},
\end{aligned}
$$

where $\Theta\left[q_{j} V+h\left(n_{j}\right)\right]$ is the pseudo-differential operator [27]

$$
\begin{aligned}
& \left(\Theta\left[q_{j} V+h\left(n_{j}\right)\right] w_{j}\right)(t, x, v) \\
& =\frac{1}{(2 \pi)^{d}} \int_{\mathbb{R}^{d}} \int_{\mathbb{R}^{d}} \frac{1}{\varepsilon}\left\{\left(q_{j} V+h\left(n_{j}\right)\right)\left(t, x+\varepsilon \frac{\eta}{2}\right)-\left(q_{j} V+h\left(n_{j}\right)\right)\left(t, x-\varepsilon \frac{\eta}{2}\right)\right\} \\
& \quad \times w_{j}\left(t, x, v^{\prime}\right) e^{i\left(v-v^{\prime}\right) \cdot \eta} d \eta d v^{\prime},
\end{aligned}
$$

and the initial datum $w_{j I}$ is the Wigner transform of the initial state $\Psi_{j I}$ given by (3). The particle densities $n_{j}=\left|\Psi_{j}\right|^{2}$, the current densities $J_{j}=\varepsilon \operatorname{Im}\left(\bar{\Psi}_{j} \nabla \Psi_{j}\right)$ and the energy densities $W_{j}=\varepsilon^{2}\left|\nabla \Psi_{j}\right|^{2}$ are given by $[6,8,18,24]$

$$
\begin{aligned}
n_{j}(t, x) & =\int_{\mathbb{R}^{d}} w_{j}(t, x, v) d v, \\
J_{j}(t, x) & =\int_{\mathbb{R}^{d}} v w_{j}(t, x, v) d v, \\
W_{j}(t, x) & =\int_{\mathbb{R}^{d}}|v|^{2} w_{j}(t, x, v) d v .
\end{aligned}
$$

Our main convergence result can be summarized as follows. Let $\left(\Psi_{1}^{\varepsilon, \lambda}, \Psi_{2}^{\varepsilon, \lambda}, V^{\varepsilon, \lambda}\right)$ be a solution to (1)-(3) and define

$$
n_{j}^{\varepsilon, \lambda}=\left|\Psi_{j}^{\varepsilon, \lambda}\right|^{2}, \quad J_{j}^{\varepsilon, \lambda}=\varepsilon \operatorname{Im}\left(\bar{\Psi}_{j}^{\varepsilon, \lambda} \nabla \Psi_{j}^{\varepsilon, \lambda}\right), \quad j=1,2 .
$$

If (i) the initial datum $\Psi_{j I}, j=1,2$, is small enough in some norm, (ii) the initial densities and velocities satisfy (11) (see Theorem 2 for a more general result), and (iii) the initial states $\left(n_{0}, u_{0}\right)$ of the Euler equation (13)-(14) are smooth enough, then the solution $\left(n_{j}^{\varepsilon, \lambda}, J_{j}^{\varepsilon, \lambda}\right), j=1,2$, converges to the solution $(n, n u)$ of the Euler equations (13)-(14) 
and the solution $w_{j}^{\varepsilon, \lambda}$ of the Wigner equation converges to a solution $w=w(t, x, v)$ of the nonlinear Vlasov equation

$$
\begin{aligned}
& \partial_{t} w+v \cdot \nabla_{x} w-\nabla_{x} h(n) \cdot \nabla_{v} w=0, \quad x \in \mathbb{R}^{d}, t>0, \\
& n(t, x)=\int_{\mathbb{R}^{d}} w(t, x, v) d v .
\end{aligned}
$$

Moreover, $w(t, x, v)=n(t, x) \delta(v-u(t, x))$ in $\mathbb{R} \times \mathbb{R}^{d} \times \mathbb{R}^{d}$. The "smallness" assumptions and the convergence results will be made precise in section 2 .

The semiclassical limit in the Schrödinger equation or in Schrödinger-Poisson systems has been done, e.g., in $[6,8,9,18,29,30]$ using the Wigner measure method of $[9,18]$. Concerning the quasineutral limit $\lambda \rightarrow 0$, there are only partial results. In particular, the limit $\lambda \rightarrow 0$ has been performed in Vlasov-Poisson systems by Brenier [2] and Grenier $[10,11,12]$, in 'linear' Schrödinger-Poisson systems by Puel [24], and in drift-diffusion equations by Gasser et al. [5, 7] and Jüngel and Peng [15]. The oscillatory behavior of the electric field is one of the main difficulties.

Recently, Brenier [2] proved the convergence of Vlasov-Poisson systems to the incompressible Euler equations if $\lambda \rightarrow 0$, where the nonnegativity of the classical phase-space distribution function is crucial to establish the convergence results. However, the Wigner function is generally not nonnegative $[18,22]$. This important difference between VlasovPoisson and Wigner-Poisson problems is one of the main difficulties to be met in dealing with asymptotic limits in Wigner-Poisson systems.

We mention that Puel [24] proved the combined semiclassical and quasineutral limit of the linear Schrödinger equation, coupled to the Poisson equation in the case of one carrier type, to the dissipative solution of the incompressible Euler equations using the modulated energy method. The originality of our result consists of the fact that we prove the convergence of the bipolar nonlinear Schrödinger-Poisson system to the compressible Euler equations.

The proof of our result is based on an estimate of the modulated energy functional

$$
\begin{aligned}
H^{\varepsilon, \lambda}(t)= & \frac{1}{2} \sum_{j=1}^{2} \int_{\mathbb{R}^{d} \times \mathbb{R}^{d}}|v-u(x, t)|^{2} w_{j}^{\varepsilon, \lambda}(t, x, v) d x d v+\frac{\lambda^{2}}{2} \int_{\mathbb{R}^{d}}\left|\nabla V^{\varepsilon, \lambda}\right|^{2} d x \\
& +a^{2} \sum_{j=1}^{2} \int_{\mathbb{R}^{d}}\left(\frac{1}{\gamma+1}\left(\left(n_{j}^{\varepsilon, \lambda}\right)^{\gamma+1}-n^{\gamma+1}\right)-n^{\gamma}\left(n_{j}^{\varepsilon, \lambda}-n\right)\right) d x,
\end{aligned}
$$

where $(n, u)$ is the smooth solution to (13)-(14). Using the fluiddynamical formulation (7)-(9) and the kinetic formulation (15) of the Schrödinger-Poisson system (1)-(2), we are able to prove that

$$
\frac{d}{d t} H^{\varepsilon, \lambda}(t) \leq C H^{\varepsilon, \lambda}(t)+C \varepsilon, \quad t>0
$$

and that $H^{\varepsilon, \lambda}(0) \rightarrow 0$ as $\varepsilon, \lambda \rightarrow 0$. This implies $H^{\varepsilon, \lambda}(t) \rightarrow 0$ and using the methods of [9] gives the result. 
This paper is organized as follows. In section 2, the precise convergence result is stated. Section 3 is devoted to the proof of the main theorem.

\section{Main result}

In this section we state our main theorem. For this, we assume that the initial datum $\left(n_{0}, u_{0}\right)$ for the Euler equations (13)-(14) satisfies

$$
n_{0}, u_{0} \in H^{s}\left(\mathbb{R}^{d}\right) \quad \text { for some } s>d / 2+2 .
$$

Then the limit equations (13)-(14) together with the corresponding initial conditions are a hyperbolic symmetrisable system. We recall the following classical result on the existence of sufficiently regular solutions for small time (see [21] for a proof).

Proposition 1 Let $\left(n_{0}, u_{0}\right)$ satisfy (22). Then there exist $T>0$, the maximal existence time, and a solution $(n, u)$ of (13)-(14) on $[0, T)$ with initial datum $\left(n_{0}, u_{0}\right)$ such that $n$, $u \in L^{\infty}\left(\left[0, T^{\prime}\right] ; H^{s}\left(\mathbb{R}^{d}\right)\right), s>d / 2+2$, for every $T^{\prime}<T$.

By Proposition 1 and Sobolev's embedding Theorem, it follows that $n, u \in L^{\infty}([0, T)$; $\left.W^{2, \infty}\left(\mathbb{R}^{d}\right)\right)$. To state our result we introduce the nonnegative ('entropy') functional

$$
\pi^{\varepsilon, \lambda}=\sum_{j=1}^{2}\left(\frac{\left(n_{j}^{\varepsilon, \lambda}\right)^{\gamma+1}-n^{\gamma+1}}{\gamma+1}-n^{\gamma}\left(n_{j}^{\varepsilon, \lambda}-n\right)\right) .
$$

Our main result reads as follows.

Theorem 2 Assume that $\left(n_{0}, u_{0}\right)$ satisfies (22), that $n_{j I}^{\varepsilon, \lambda}=\left|\Psi_{j I}^{\varepsilon, \lambda}\right|^{2}, j=1,2$, satisfies $n_{1 I}^{\varepsilon, \lambda}=n_{2 I}^{\varepsilon, \lambda}=n_{0} \in L^{\gamma+1}\left(\mathbb{R}^{d}\right)$, that $\left\|\Psi_{j I}^{\varepsilon, \lambda}\right\|_{H^{s}}$ is sufficiently small, and finally that

$$
\left\|u_{0} \Psi_{j I}^{\varepsilon, \lambda}+i \varepsilon \nabla \Psi_{j I}^{\varepsilon, \lambda}\right\|_{L^{2}} \rightarrow 0 \quad \text { as } \varepsilon \rightarrow 0, \lambda \rightarrow 0, j=1,2 .
$$

Then, for $j=1,2$, as $\varepsilon \rightarrow 0$ and $\lambda \rightarrow 0$,

$$
\begin{aligned}
n_{j}^{\varepsilon, \lambda} \rightarrow n & \text { weakly }{ }^{*} \text { in } L^{\infty}\left(0, T ; L^{\gamma+1}\left(\mathbb{R}^{d}\right)\right), \\
\pi^{\varepsilon, \lambda} \rightarrow 0 & \text { strongly in } L^{\infty}\left(0, T ; L^{1}\left(\mathbb{R}^{d}\right)\right), \\
J_{j}^{\varepsilon, \lambda} \rightarrow n u & \text { in the sense of } L^{\infty}\left(0, T ;\left(\mathcal{M}\left(\mathbb{R}^{d}\right)\right)^{d}\right), \\
w_{j}^{\varepsilon, \lambda} \rightarrow w & \text { in the sense of } L^{\infty}\left(0, T ; \mathcal{A}^{\prime} \omega-*\right) \omega-*,
\end{aligned}
$$

where $n_{j}^{\varepsilon, \lambda}$ and $J_{j}^{\varepsilon, \lambda}$ are defined as in (19), $w(t, x, v)=n(t, x) \delta(v-u(t, x)),(n, u)$ is the unique smooth solution to the compressible Euler equations (13)-(14) with initial datum $\left(n_{0}, u_{0}\right)$, and $w \in C^{0,1}\left([0, T) ; H^{-s}\left(\mathbb{R}^{d} \times \mathbb{R}^{d}\right)\right)$ solves the nonlinear Vlasov equation (20) in the sense of distributions (see [19]). 
Furthermore, $T>0$ is the maximal existence time defined by Proposition 1, $\mathcal{M}\left(\mathbb{R}^{d}\right)$ is the Radon measure space on $\mathbb{R}^{d}$, and $\mathcal{A}$ is the (separable) Banach algebra of test functions

$$
\mathcal{A}=\left\{\phi \in C^{0}\left(\mathbb{R}_{x}^{d} \times \mathbb{R}_{v}^{d}\right) \mid\left(\mathcal{F}_{v \rightarrow \eta} \phi\right)(x, \eta) \in L^{1}\left(\mathbb{R}_{\eta}^{d} ; C^{0}\left(\mathbb{R}_{x}^{d}\right)\right)\right\}
$$

equipped with the norm

$$
\|\phi\|_{\mathcal{A}}:=\int_{\mathbb{R}_{\eta}^{d}} \sup _{x}\left|\mathcal{F}_{v \rightarrow \eta} \phi(x, \eta)\right| d \eta .
$$

The symbol $\mathcal{F}_{v \rightarrow \eta}$ denotes the Fourier transform in $v$ and $\omega-*$ is the convergence in the weak* topology.

Remark 3 The smallness and smoothness of the initial datum guarantee the existence of a global small solution to the Schrödinger-Poisson system, see Lemma 5 below or [13]. For different conditions which guarantee the global existence of smooth solutions to nonlinear Schrödinger systems, we refer to [1]. For the definition of the Banach algebra $\mathcal{A}$, see [18].

Remark 4 In order to understand the assumption (24) notice that the condition $n_{1 I}^{\varepsilon, \lambda}=$ $n_{2 I}^{\varepsilon, \lambda}=n_{0}$ implies (see (33))

$$
\begin{aligned}
H^{\varepsilon, \lambda}(0) & =\frac{1}{2} \sum_{j=1}^{2} \int_{\mathbb{R}^{d} \times \mathbb{R}^{d}}|v-u(t, x)|^{2} w_{j I}^{\varepsilon, \lambda}(t, x, v) d x d v \\
& =\left\|u_{0} \Psi_{j I}^{\varepsilon, \lambda}+i \varepsilon \nabla \Psi_{j I}^{\varepsilon, \lambda}\right\|_{L^{2}}^{2} .
\end{aligned}
$$

Thus (24) means that the modulated energy at $t=0$ vanishes in the limit $\varepsilon, \lambda \rightarrow 0$. Moreover, if the wave function satisfies

$$
\Psi_{j}^{\varepsilon, \lambda}(x, t)=\sqrt{n_{j}^{\varepsilon, \lambda}(x, t)} \exp \left(i S_{j}^{\varepsilon, \lambda}(x, t) / \varepsilon\right), \quad j=1,2
$$

then it holds

$$
\left\|u_{0} \Psi_{j I}^{\varepsilon, \lambda}+i \varepsilon \nabla \Psi_{j I}^{\varepsilon, \lambda}\right\|_{L^{2}}^{2}=\varepsilon^{2}\left\|\nabla \sqrt{n_{j I}^{\varepsilon, \lambda}}\right\|_{L^{2}}^{2}+\left\|\sqrt{n_{j I}^{\varepsilon, \lambda}}\left(u_{0}-\nabla S_{j I}^{\varepsilon, \lambda}\right)\right\|_{L^{2}}^{2} .
$$

In particular, if $n_{j I}^{\varepsilon, \lambda}=n_{0}$ and $\nabla S_{j I}^{\varepsilon, \lambda}=u_{0}, j=1,2$, then

$$
\varepsilon\left\|\nabla \sqrt{n_{0}}\right\|_{L^{2}}=\varepsilon\left\|\nabla \sqrt{n_{j I}^{\varepsilon, \lambda}}\right\|_{L^{2}}=\left\|u_{0} \Psi_{j I}^{\varepsilon, \lambda}+i \varepsilon \nabla \Psi_{j I}^{\varepsilon, \lambda}\right\|_{L^{2}} .
$$

\section{Proof of Theorem 2}

In this section, we will prove our main theorem by the Wigner measure method. We divide the proof into several steps. 


\subsection{Standard energy estimates}

Lemma 5 Under the assumptions of Theorem 2, for any fixed $\varepsilon, \lambda,(1)-(3)$ has a unique global smooth small solution $\Psi_{j}^{\varepsilon, \lambda}, V^{\varepsilon, \lambda} \in L^{\infty}\left(\left[0, T^{\prime}\right], H^{s}\left(\mathbb{R}^{d}\right)\right), j=1,2, s>d / 2+2$, for any $T^{\prime}<\infty$. Moreover, we have the following estimates, for $t>0, j=1,2$ :

$$
\begin{aligned}
& \left\|\Psi_{j}^{\varepsilon, \lambda}(t, \cdot)\right\|_{L^{2}}^{2}=\left\|n_{j I}^{\varepsilon, \lambda}(\cdot)\right\|_{L^{1}}, \\
& \frac{d}{d t}\left\{\varepsilon^{2} \sum_{j=1}^{2}\left\|\nabla \Psi_{j}^{\varepsilon, \lambda}(t, \cdot)\right\|_{L^{2}}^{2}+\lambda^{2}\left\|\nabla V^{\varepsilon, \lambda}(t, \cdot)\right\|_{L^{2}}^{2}+2 \sum_{j=1}^{2} \int_{\mathbb{R}^{d}} \int_{0}^{n_{j}^{\varepsilon, \lambda}} h(s) d s d x\right\}=0 .
\end{aligned}
$$

Proof. The existence and uniqueness of small smooth solutions can be obtained by modifying the proof of $[3,13]$. We omit the details.

Now we establish the a priori estimates. Multiply (1) with $\bar{\Psi}_{j}^{\varepsilon, \lambda}$, integrate with respect to $x$ and integrate by parts to obtain

$$
\varepsilon i \int \partial_{t} \Psi_{j}^{\varepsilon, \lambda} \overline{\Psi_{j}^{\varepsilon, \lambda}} d x=\frac{\varepsilon^{2}}{2} \int \nabla \Psi_{j}^{\varepsilon, \lambda} \cdot \nabla \overline{\Psi_{j}^{\varepsilon, \lambda}} d x+\int\left(q_{j} V^{\varepsilon, \lambda}+h\left(n_{j}^{\varepsilon, \lambda}\right)\right)\left|\Psi_{j}^{\varepsilon, \lambda}\right|^{2} d x .
$$

Here and in the following, we omit the integration domain $\mathbb{R}^{d}$ in the integral symbols. The imaginary part of (27) yields

$$
\frac{1}{2} \frac{d}{d t} \int\left|\Psi_{j}^{\varepsilon, \lambda}\right|^{2} d x=0, \quad j=1,2,
$$

and hence (25).

Multiplying (1) with $\partial_{t} \bar{\Psi}_{j}^{\varepsilon, \lambda}$, integrating with respect to $x$ and integrating by parts gives for $j=1,2$

$$
i \varepsilon \int\left|\partial_{t} \Psi_{j}^{\varepsilon, \lambda}\right|^{2} d x=\frac{\varepsilon^{2}}{2} \int \nabla \Psi_{j}^{\varepsilon, \lambda} \partial_{t} \nabla \overline{\Psi^{\varepsilon, \lambda}} j d x+\int\left(q_{j} V^{\varepsilon, \lambda}+h\left(n_{j}^{\varepsilon, \lambda}\right)\right) \Psi_{j}^{\varepsilon, \lambda} \partial_{t} \bar{\Psi}^{\varepsilon, \lambda}{ }_{j} d x .
$$

Taking the sum of the real part of this equation for $j=1,2$ and using $q_{1}=1=-q_{2}$, we get

$$
\begin{aligned}
\frac{\varepsilon^{2}}{2} \frac{d}{d t} \int \sum_{j=1}^{2}\left|\nabla \Psi_{j}^{\varepsilon, \lambda}\right|^{2} d x & =-\int\left(V^{\varepsilon, \lambda} \partial_{t}\left(\left|\Psi_{1}^{\varepsilon, \lambda}\right|^{2}-\left|\Psi_{2}^{\varepsilon, \lambda}\right|^{2}\right)+\sum_{j=1}^{2} h\left(n_{j}^{\varepsilon, \lambda}\right) \partial_{t}\left|\Psi_{j}^{\varepsilon, \lambda}\right|^{2}\right) d x \\
& =\lambda^{2} \int V^{\varepsilon, \lambda} \partial_{t} \Delta V^{\varepsilon, \lambda} d x-\int \sum_{j=1}^{2} h\left(n_{j}^{\varepsilon, \lambda}\right) \partial_{t} n_{j}^{\varepsilon, \lambda} d x \\
& =-\frac{\lambda^{2}}{2} \frac{d}{d t} \int\left|\nabla V^{\varepsilon, \lambda}\right|^{2} d x-\frac{d}{d t} \int \sum_{j=1}^{2} \int_{0}^{n_{j}^{\varepsilon, \lambda}} h(s) d s d x
\end{aligned}
$$

where we have used the Poisson equation (2). This gives the estimate (26). 
Lemma 6 (Quasi-neutrality) It holds, as $\varepsilon, \lambda \rightarrow 0$,

$$
n_{1}^{\varepsilon, \lambda}-n_{2}^{\varepsilon, \lambda} \rightarrow 0 \quad \text { in } \mathcal{D}^{\prime}\left(\left(0, T^{\prime}\right) \times \mathbb{R}^{d}\right), T^{\prime}<\infty .
$$

Proof. Use $\phi \in \mathcal{D}\left(\left(0, T^{\prime}\right) \times \mathbb{R}^{d}\right)$ as a test function in the Poisson equation:

$$
\begin{aligned}
\int\left(n_{1}^{\varepsilon, \lambda}-n_{2}^{\varepsilon, \lambda}\right) \phi d x d t & =-\lambda^{2} \int \Delta V^{\varepsilon, \lambda} \cdot \phi d x d t \\
& =\lambda^{2} \int \nabla V^{\varepsilon, \lambda} \cdot \nabla \phi d x d t \\
& \leq \lambda^{2}\left\|\nabla V^{\varepsilon, \lambda}\right\|_{L^{2}}\|\nabla \phi\|_{L^{2}} \\
& \leq C \lambda \\
& \rightarrow 0 \quad(\lambda \rightarrow 0),
\end{aligned}
$$

using Lemma 5.

From the above proof follows immediately that the convergence (29) holds in the space $L^{\infty}\left(0, T^{\prime} ; H^{1}\left(\mathbb{R}^{d}\right)\right)$.

\subsection{Conservation law formulation and weak convergence}

Lemma 7 The variables $n_{j}^{\varepsilon, \lambda}$ and $J_{j}^{\varepsilon, \lambda}, j=1,2$, defined in (19), satisfy the equations

$$
\begin{aligned}
\partial_{t} n_{j}^{\varepsilon, \lambda}+\operatorname{div} J_{j}^{\varepsilon, \lambda} & =0, \\
\partial_{t} J_{j}^{\varepsilon, \lambda}+\nabla_{x} \cdot \int(v \otimes v) w_{j}^{\varepsilon, \lambda} d v+n_{j}^{\varepsilon, \lambda} q_{j} \nabla V^{\varepsilon, \lambda}+n_{j}^{\varepsilon, \lambda} \nabla h\left(n_{j}^{\varepsilon, \lambda}\right) & =0,
\end{aligned}
$$

and the energy estimate

$$
\frac{d}{d t}\left\{\int \sum_{j=1}^{2}|v|^{2} w_{j}^{\varepsilon, \lambda} d x d v+\lambda^{2} \int\left|\nabla V^{\varepsilon, \lambda}\right|^{2} d x+2 \int \sum_{j=1}^{2} \int_{0}^{n_{j}^{\varepsilon, \lambda}} h(s) d s d x\right\}=0 .
$$

Proof. The moment equations (30)-(31) are easily obtained from the Wigner equation, see $[6,8,18,29]$, and the total energy conservation (32) can be obtained from (18) and (26).

Lemma 8 Let $w_{j}^{\varepsilon, \lambda}(t, x, v)$ be the Wigner transform of $\Psi_{j}^{\varepsilon, \lambda}(t, x)$. Then we have, maybe after extracting subsequences,

$$
\begin{aligned}
w_{j}^{\varepsilon, \lambda} \rightarrow w_{j} & \text { weakly*in } L^{\infty}\left(\mathbb{R}_{t}, \mathcal{A}^{\prime} \omega-*\right), \\
n_{j}^{\varepsilon, \lambda} \rightarrow n_{j}:=\int w_{j}(t, x, d v) & \text { in } C_{b}\left(\mathbb{R}_{t} ; \mathcal{M}^{+}\left(\mathbb{R}_{x}^{d}\right) \omega-*\right), \\
J_{j}^{\varepsilon, \lambda} \rightarrow J_{i}:=\int v w_{j}(t, x, d v) & \text { in } C_{b}\left(\mathbb{R}_{t} ; \mathcal{M}\left(\mathbb{R}_{x}^{d}\right) \omega-*\right)
\end{aligned}
$$


uniformly on compact subsets of $t \in \mathbb{R}$, in $\left(C_{0}\left(\mathbb{R}_{x}^{d}\right)\right)^{\prime} \omega-*$, and in $\left(\left(C_{0}\left(\mathbb{R}_{x}^{d}\right)\right)^{\prime}\right)^{d} \omega-*$, respectively. Moreover, $w_{j}(t, x, v)$ is a nonnegative Radon measure on $\mathbb{R}^{d} \times \mathbb{R}^{d}$ for any fixed time $t$.

For the proof of Lemma 8 , we refer to $[8,18]$. The space $C_{b}\left(\mathbb{R}_{t} ; X\right)$ denotes the space of bounded continuous functions $\mathbb{R} \rightarrow X$ and $\mathcal{M}^{+}\left(\mathbb{R}^{d}\right)$ the space of nonnegative Radon measures on $\mathbb{R}^{d}$.

Remark 9 The function $w_{j}$ in Lemma 8 is called the Wigner measure of $\Psi_{j}^{\varepsilon, \lambda}$. It has a very close relation with the H-measure of $\Psi_{j}^{\varepsilon, \lambda}$ (see $[18,26]$ ).

In the following, we will prove that the nonnegative Radon measure $w_{j}(t, x, v), j=1,2$, is in fact a delta distribution, i.e.

$$
w_{1}(t, x, v)=w_{2}(t, x, v)=n(t, x) \delta(v-u(t, x)),
$$

where $(n, u)$ is the smooth solution to the compressible Euler equations (13)-(14) with initial datum $\left(n_{0}, u_{0}\right)$ defined on $[0, T)$, where $T$ is the maximal existence time.

This idea has been successfully used by several authors, in particular, by Evans [4] for the global existence of weak solutions to conservation laws, by Xin and Zhang [28] for the global existence of weak solutions to shallow water wave equations, by Zhang, Zheng, Mauser [30] and Zhang [29] for the semi-classical limit of the Schrödinger-Poisson equations and finally, by Lin and Zhang [20] for the hydrodynamic limit of GinzburgLandau vortices.

\subsection{Modulated energy estimates}

We use the modulated energy functional (21) defined in the introduction. The functional is nonnegative since the function in the last integral in (21) is convex. We notice the following relation between the Wigner transform and the wave function:

$$
\int_{\mathbb{R}^{d}} \int_{\mathbb{R}^{d}}|v-u(t, x)|^{2} w_{j}^{\varepsilon, \lambda}(t, x, v) d x d v=\int\left|u \Psi_{j}^{\varepsilon, \lambda}+i \varepsilon \nabla \Psi_{j}^{\varepsilon, \lambda}\right|^{2}(t, x) d x, \quad t>0 .
$$

In fact, by (16)-(18), we have, for $j=1,2$,

$$
\begin{aligned}
& \int_{\mathbb{R}^{d}} \int_{\mathbb{R}^{d}}|v-u(t, x)|^{2} w_{j}^{\varepsilon, \lambda}(t, x, v) d x d v \\
& \quad=\int_{\mathbb{R}^{d}} \int_{\mathbb{R}^{d}}|v|^{2} w_{j}^{\varepsilon, \lambda}(t, x, v) d x d v+\int|u|^{2}\left|\Psi_{j}^{\varepsilon, \lambda}\right|^{2} d x-2 \int u \cdot J_{j}^{\varepsilon, \lambda} d x \\
& =\int\left\{\varepsilon^{2}\left|\nabla \Psi_{j}^{\varepsilon, \lambda}\right|^{2}-2 \varepsilon \operatorname{Im}\left(\overline{\Psi_{j}^{\varepsilon, \lambda}} \nabla \Psi_{j}^{\varepsilon, \lambda}\right) u+|u|^{2}\left|\Psi_{j}^{\varepsilon, \lambda}\right|^{2}\right\} d x \\
& \quad=\int\left|u \Psi_{j}^{\varepsilon, \lambda}+i \varepsilon \nabla \Psi_{j}^{\varepsilon, \lambda}\right|^{2} d x,
\end{aligned}
$$


which yields (33).

We have the following convergence result for $H^{\varepsilon, \lambda}$ which is the main step of the proof of Theorem 2.

Lemma 10 Let $(n, u)$ be the smooth solution of the compressible Euler equation (13)-(14) on $[0, T) \times \mathbb{R}^{d}$ where $T>0$ is the maximal existence time. Then we have, for any $t \in[0, T)$,

$$
H^{\varepsilon, \lambda}(t) \rightarrow 0 \quad \text { as } \varepsilon, \lambda \rightarrow 0 .
$$

Proof. Using the definition of $H^{\varepsilon, \lambda}$ and the conservation of the total energy (32), we have, for the particle density $n_{j}^{\varepsilon, \lambda}$ and the current density $J_{j}^{\varepsilon, \lambda}$,

$$
\begin{aligned}
\frac{d}{d t} H^{\varepsilon, \lambda}(t)= & \sum_{j=1}^{2} \frac{d}{d t} \int \frac{1}{2}|u(t, x)|^{2} n_{j}^{\varepsilon, \lambda}(t, x) d x-\sum_{j=1}^{2} \int \partial_{t}\left(u(t, x) \cdot J_{j}^{\varepsilon, \lambda}(t, x)\right) d x \\
& -a^{2} \sum_{j=1}^{2} \frac{d}{d t} \int n^{\gamma}(t, x) n_{j}^{\varepsilon, \lambda}(t, x) d x+\frac{2 a^{2} \gamma}{\gamma+1} \frac{d}{d t} \int n^{\gamma+1}(t, x) d x
\end{aligned}
$$

We compute the right-hand side term by term. Using (30)-(31), we get

$$
\begin{aligned}
\frac{d}{d t} \int \frac{1}{2}|u(t, x)|^{2} n_{j}^{\varepsilon, \lambda}(t, x) d x & =\int\left\{u\left(\partial_{t} u\right) n_{j}^{\varepsilon, \lambda}+\frac{1}{2}|u|^{2} \partial_{t} n_{j}^{\varepsilon, \lambda}\right\} d x \\
& =\int\left\{u\left(\partial_{t} u\right) n_{j}^{\varepsilon, \lambda}-\frac{1}{2}|u|^{2} \operatorname{div} J_{j}^{\varepsilon, \lambda}\right\} d x \\
& =\int\left\{-n_{j}^{\varepsilon, \lambda} u(u \cdot \nabla u+\nabla h(n))+\frac{1}{2} J_{j}^{\varepsilon, \lambda} \cdot \nabla|u|^{2}\right\} d x .
\end{aligned}
$$

The second term on the right-hand side of (34) can be written as

$$
\begin{aligned}
- & \int \partial_{t}\left(u(t, x) \cdot J_{j}^{\varepsilon, \lambda}(t, x)\right) d x \\
= & \int\left(-u \partial_{t} J_{j}^{\varepsilon, \lambda}-J_{j}^{\varepsilon, \lambda} \partial_{t} u\right) d x \\
= & \int\left\{\left(\nabla_{x} \cdot \int_{\mathbb{R}_{v}}(v \otimes v) w_{j}^{\varepsilon, \lambda} d v+q_{j} \nabla V^{\varepsilon, \lambda} n_{j}^{\varepsilon, \lambda}+n_{j}^{\varepsilon, \lambda} \nabla h\left(n_{j}^{\varepsilon, \lambda}\right)\right) u\right. \\
& \left.+J_{j}^{\varepsilon, \lambda}(u \cdot \nabla u+\nabla h(n))\right\} d x \\
= & -\int D u: \int_{\mathbb{R}_{v}}(v \otimes v) w_{j}^{\varepsilon, \lambda} d v d x+q_{j} \int \nabla V^{\varepsilon, \lambda} n_{j}^{\varepsilon, \lambda} u d x \\
& +\int n_{j}^{\varepsilon, \lambda} u \nabla h\left(n_{j}^{\varepsilon, \lambda}\right) d x+\int J_{j}^{\varepsilon, \lambda}(u \cdot \nabla u+\nabla h(n)) d x,
\end{aligned}
$$


where $D u$ denotes the matrix $\left(\partial u_{j} / \partial x_{k}\right)_{j k}$ and the symbol ":" means summation over both matrix indices. Furthermore, we obtain

$$
\begin{aligned}
\frac{d}{d t} \int n^{\gamma} n_{j}^{\varepsilon, \lambda} d x & =\int\left(\gamma n^{\gamma-1}\left(\partial_{t} n\right) n_{j}^{\varepsilon, \lambda}+n^{\gamma} \partial_{t} n_{j}^{\varepsilon, \lambda}\right) d x \\
& =-\int\left(\gamma n^{\gamma-1} \operatorname{div}(n u) n_{j}^{\varepsilon, \lambda}+n^{\gamma} \operatorname{div} J_{j}^{\varepsilon, \lambda}\right) d x \\
& =\int\left(\gamma n^{\gamma} u \nabla n_{j}^{\varepsilon, \lambda}+(\gamma-1) \nabla n^{\gamma} n_{j}^{\varepsilon, \lambda} u+J_{j}^{\varepsilon, \lambda} \nabla n^{\gamma}\right) d x
\end{aligned}
$$

and

$$
\begin{aligned}
\frac{d}{d t} \int n^{\gamma+1} d x & =(\gamma+1) \int n^{\gamma} \partial_{t} n d x \\
& =-(\gamma+1) \int n^{\gamma} \operatorname{div}(n u) d x \\
& =(\gamma+1) \int n u \nabla n^{\gamma} d x
\end{aligned}
$$

Substituting (35)-(38) into (34) and using the definitions (4) of $h$ and (23) of $\pi^{\varepsilon, \lambda}$, we have

$$
\begin{aligned}
\frac{d}{d t} H^{\varepsilon, \lambda}(t)= & -\sum_{j=1}^{2} \int D u: \int(v \otimes v) w_{j}^{\varepsilon, \lambda}(t, x, v) d v d x+\int\left(n_{1}^{\varepsilon, \lambda}-n_{2}^{\varepsilon, \lambda}\right) u \nabla V^{\varepsilon, \lambda} d x \\
& -\sum_{j=1}^{2} \int\left(n_{j}^{\varepsilon, \lambda} u(u \cdot \nabla u)-D u:\left(J_{j}^{\varepsilon, \lambda} \otimes u\right)-D u:\left(u \otimes J_{j}^{\varepsilon, \lambda}\right)\right) d x \\
& +a^{2} \sum_{j=1}^{2} \int\left\{n_{j}^{\varepsilon, \lambda} u \nabla\left(n_{j}^{\varepsilon, \lambda}\right)^{\gamma}-n_{j}^{\varepsilon, \lambda} u \nabla n^{\gamma}-\gamma n^{\gamma} u \nabla n_{j}^{\varepsilon, \lambda}\right. \\
& \left.-(\gamma-1) \nabla n^{\gamma} n_{j}^{\varepsilon, \lambda} u+\gamma n u \nabla n^{\gamma}\right\} d x \\
= & -\sum_{j=1}^{2} \int D u: \int(v-u) \otimes(v-u) w_{j}^{\varepsilon, \lambda}(t, x, d v) d x \\
& -a^{2} \gamma \int \pi^{\varepsilon, \lambda} \operatorname{div} u d x+\int\left(n_{1}^{\varepsilon, \lambda}-n_{2}^{\varepsilon, \lambda}\right) u \nabla V^{\varepsilon, \lambda} d x
\end{aligned}
$$

Elementary calculations, by using the relation between the Wigner transform and the wave 
function again, lead to

$$
\begin{aligned}
& \left|-\int D u: \int(v-u) \otimes(v-u) w_{j}^{\varepsilon, \lambda}(t, x, d v) d x\right| \\
& =\mid \int \sum_{k, l=1}^{d}\left\{\frac{\partial u_{l}}{\partial x_{k}} \operatorname{Re}\left[\left(u_{k}+i \varepsilon \frac{\partial}{\partial x_{k}}\right) \Psi_{j}^{\varepsilon, \lambda} \overline{\left(u_{l}+i \varepsilon \frac{\partial}{\partial x_{l}}\right) \Psi_{j}^{\varepsilon, \lambda}}\right) d x\right. \\
& \left.\quad+\frac{\varepsilon^{2}}{2} \int \frac{\partial^{2} u_{l}}{\partial x_{k}} \operatorname{Re}\left(\overline{\Psi_{j}^{\varepsilon, \lambda}} \frac{\partial}{\partial x_{l}} \Psi_{j}^{\varepsilon, \lambda}\right)\right\} d x \mid \\
& \leq \quad C \int\left|\left(u+i \varepsilon \nabla_{x}\right) \Psi_{j}^{\varepsilon, \lambda}\right|^{2} d x+C \varepsilon\left\|D_{x}^{2} u\right\|_{L^{\infty}\left\|\Psi_{j}^{\varepsilon, \lambda}\right\|_{L^{2}}\left\|\varepsilon \nabla \Psi_{j}^{\varepsilon, \lambda}\right\|_{L^{2}} .}
\end{aligned}
$$

Here and in the following, $C>0$ denote generic constants only depending on $T$ and $\|u\|_{W^{2, \infty}}$. Using the Poisson equation (9), we have

$$
\begin{aligned}
\int\left(n_{1}^{\varepsilon, \lambda}-n_{2}^{\varepsilon, \lambda}\right) u \nabla V^{\varepsilon, \lambda} d x & =-\int \lambda^{2} \Delta V^{\varepsilon, \lambda} u \nabla V^{\varepsilon, \lambda} d x \\
& =\lambda^{2} \int D u:\left(\nabla V^{\varepsilon, \lambda} \otimes \nabla V^{\varepsilon, \lambda}\right) d x-\frac{\lambda^{2}}{2} \int\left|\nabla V^{\varepsilon, \lambda}\right|^{2} \operatorname{div} u d x \\
& \leq C \lambda^{2} \int\left|\nabla V^{\varepsilon, \lambda}\right|^{2} d x
\end{aligned}
$$

Thus, from (39) together with (25), (26), (33), (40), and (41), we conclude

$$
\frac{d}{d t} H^{\varepsilon, \lambda}(t) \leq C H^{\varepsilon, \lambda}(t)+C \varepsilon .
$$

The assumption on the initial data yields

$$
\begin{aligned}
H^{\varepsilon, \lambda}(0)= & \frac{1}{2} \sum_{j=1}^{2} \iint\left|v-u_{0}\right|^{2} w_{j}^{\varepsilon, \lambda}(0, x, v) d v d x+\frac{\lambda^{2}}{2} \int\left|\nabla V^{\varepsilon, \lambda}(0, x)\right|^{2} d x \\
& +a^{2} \int \pi^{\varepsilon, \lambda}(0, x) d x \\
= & \frac{1}{2} \sum_{j=1}^{2} \int\left|\left(u+i \varepsilon \nabla_{x}\right) \Psi_{j}^{\varepsilon, \lambda}(0, x)\right|^{2} d x \\
\rightarrow & 0 \quad \text { as } \varepsilon, \lambda \rightarrow 0 .
\end{aligned}
$$

Hence we obtain from (42) and (43), employing Gronwall's inequality,

$$
\lim _{\varepsilon \rightarrow 0, \lambda \rightarrow 0} H^{\varepsilon, \lambda}(t)=0 .
$$

This completes the proof of Lemma 10 . 
Lemma 11 For any smooth cut-off function $\sigma(x, v)$ with

$$
\sigma(x, v)=1 \quad \text { for }|x|+|v| \leq \frac{1}{2}, \quad \operatorname{supp} \sigma \subset B(0,1)
$$

there exists a positive constant $M=M(\sigma, u)$ such that

$$
\begin{gathered}
\int \sigma^{2}(x / R, v / R)|v-u(t, x)|^{2} w_{j}^{\varepsilon, \lambda}(t, x, v) d v d x \\
\leq M\left\|\left(u(t, \cdot)+i \varepsilon \nabla_{x}\right) \Psi_{j}^{\varepsilon, \lambda}(t, \cdot)\right\|_{L^{2}}^{2}+r_{\varepsilon},
\end{gathered}
$$

where $r_{\varepsilon} \leq \varepsilon M(\sigma, u)\left\|\Psi_{j}^{\varepsilon, \lambda}(t, \cdot)\right\|_{L^{2}}$.

Proof. For the proof, see $[9,29]$. In fact, it is a direct consequence of Proposition 1.1 in [9] or of the formulas (3.31)-(3.32) in [29].

\subsection{End of the proof of Theorem 2}

First, by (26) and Lemmas 6 and 10 , we get the convergence of $n_{j}^{\varepsilon, \lambda} \rightarrow n$ in $L^{\infty}(0, T$; $\left.L^{\gamma+1}\left(\mathbb{R}^{d}\right)\right)$ weakly*.

We prove that $w_{1}(t, x, v)=w_{2}(t, x, v)=w(t, x, v)=n(t, x) \delta(v-u(t, x))$ and hence $J(t, x)=J_{1}(t, x)=J_{2}(t, x)=\int v w(t, x, v) d v=n u(t, x)$ for $t \in(0, T), x, v \in \mathbb{R}^{d}$. To this end, we take a smooth cut-off function $\sigma(x, v)$ satisfying (44). Then by using Lemmas 8-11, we have for $t \in(0, T)$,

$$
\begin{aligned}
\int & \sigma^{2}(x / R, v / R)|v-u(t, x)|^{2} w_{j}(t, x, v) d v d x \\
& =\lim _{\varepsilon \rightarrow 0, \lambda \rightarrow 0} \int \sigma^{2}(x / R, v / R)|v-u(t, x)|^{2} w_{j}^{\varepsilon, \lambda}(t, x, v) d v d x \\
\leq & M \lim _{\varepsilon \rightarrow 0, \lambda \rightarrow 0}\left\|\left(u(t, \cdot)+i \varepsilon \nabla_{x}\right) \Psi_{j}^{\varepsilon, \lambda}(t, \cdot)\right\|_{L^{2}}^{2} \\
\leq & C M \lim _{\varepsilon \rightarrow 0, \lambda \rightarrow 0} H^{\varepsilon, \lambda}(t) \\
& =0 .
\end{aligned}
$$

Noticing that $w_{j}(t, \cdot, \cdot) \in \mathcal{M}^{+}\left(\mathbb{R}^{d} \times \mathbb{R}^{d}\right)$, Fatou's Lemma implies

$$
\int|v-u(t, x)|^{2} w_{j}(t, x, v) d x d v=0
$$

from which we find that there is a non-negative Radon measure $\nu_{t}(x)$ such that

$$
w_{j}(t, x, v)=\nu_{t}(x) \otimes \delta(v-u(t, x)) .
$$


Using Lemma 8 and the weak convergence of $n_{j}^{\varepsilon, \lambda}$, we have

$$
\int w_{j}(t, x, v) d v=n(t, x)
$$

These two identities yield

$$
w_{j}(t, x, v)=n(t, x) \delta(v-u(t, x)), \quad j=1,2 .
$$

The above argument shows that we do not need to take a subsequence of $w_{j}^{\varepsilon, \lambda}(t, x, v)$ since the smooth solution of (13)-(14) is unique.

Now, we prove that $w \in C^{0,1}\left([0, T) ; H^{-s}\left(\mathbb{R}^{d} \times \mathbb{R}^{d}\right)\right)$. In fact, for any test function $\phi \in C_{0}^{\infty}\left(\mathbb{R}_{x} \times \mathbb{R}_{v}\right)$, we have (with a slight abuse of notation)

$$
\begin{aligned}
\frac{d}{d t} \int_{\mathbb{R}_{x}^{d}} \int_{\mathbb{R}_{v}^{d}} w(t, x, v) \phi(x, v) d x d v & =\frac{d}{d t} \int_{\mathbb{R}_{x}^{d}} \int_{\mathbb{R}_{v}^{d}} n(t, x) \delta(v-u(t, x)) \phi(x, v) d x d v \\
& =\frac{d}{d t} \int_{\mathbb{R}_{x}^{d}} n(t, x) \phi(x, u(t, x)) d x \\
& =\int_{\mathbb{R}_{x}^{d}}\left(\left(\partial_{t} n\right)(t, x) \phi(x, u(t, x))+n(t, x) \nabla_{v} \phi \partial_{t} u\right) d x .
\end{aligned}
$$

Hence, for $s>d / 2+2$,

$$
\begin{aligned}
\left|\frac{d}{d t} \int_{\mathbb{R}_{x}^{d}} \int_{\mathbb{R}_{v}^{d}} w(t, x, v) \phi(x, v) d x d v\right| & \leq\left(\|n\|_{L^{2}}\left\|\partial_{t} u\right\|_{L^{2}}+\left\|\partial_{t} n\right\|_{L^{2}}\right)\|\phi\|_{W^{1, \infty}} \\
& \leq C\|\phi\|_{H^{s}}
\end{aligned}
$$

which gives $w \in C^{0,1}\left([0, T) ; H^{-s}\left(\mathbb{R}^{d} \times \mathbb{R}^{d}\right)\right)$.

Finally, we verify that $w(t, x, v)=n(t, x) \delta(v-u(t, x))$ is a solution of (20) in the sense of distributions. To this end, it suffices to prove that

$$
\int_{0}^{T} \int_{\mathbb{R}_{x}^{d}} n(t, x)\left\{\partial_{t} \phi(t, x, u)+u \nabla_{x} \phi(t, x, u)-\nabla_{x} h(n) \nabla_{u} \phi(t, x, u)\right\} d x d t=0
$$

holds for any test function $\phi \in C_{0}^{\infty}\left((0, T) \times \mathbb{R}^{d} \times \mathbb{R}^{d}\right)$, and hence

$$
\int_{0}^{T} \int_{\mathbb{R}_{x}^{d}} \int_{\mathbb{R}_{v}^{d}} w(t, x, v)\left\{\partial_{t} \phi(t, x, v)+v \nabla_{x} \phi(t, x, v)-\nabla_{x} h(n) \nabla_{v} \phi(t, x, v)\right\} d x d v d t=0 .
$$

In fact, since $(n, u)$ is the smooth solution of the Euler equation (13)-(14), we have, for 
any test function $\phi \in C_{0}^{\infty}\left((0, T) \times \mathbb{R}^{d} \times \mathbb{R}^{d}\right)$,

$$
\begin{aligned}
0= & \int_{0}^{T} \int_{\mathbb{R}_{x}^{d}}\left(\partial_{t} n(t, x)+\operatorname{div}(n u)(t, x)\right) \phi(t, x, u(t, x)) d x d t \\
= & -\int_{0}^{T} \int_{\mathbb{R}_{x}^{d}} n(t, x)\left\{\partial_{t} \phi(t, x, u)+\nabla_{u} \phi(t, x, u) u_{t}+u \nabla_{x} \phi(t, x, u)\right. \\
& \left.+u \nabla_{x} u \nabla_{u} \phi(t, x, u)\right\} d x d t \\
= & -\int_{0}^{T} \int_{\mathbb{R}_{x}^{d}} n(t, x)\left\{\partial_{t} \phi(t, x, u)+u \nabla_{x} \phi(t, x, u)+\left(\partial_{t} u+\left(u \cdot \nabla_{x}\right) u\right) \nabla_{u} \phi(t, x, u)\right\} d x d t \\
= & -\int_{0}^{T} \int_{\mathbb{R}_{x}^{d}} n(t, x)\left\{\partial_{t} \phi(t, x, u)+u \nabla_{x} \phi(t, x, u)-\nabla_{x} h(n) \nabla_{u} \phi(t, x, u)\right\} d x d t,
\end{aligned}
$$

which gives (46). This proves Theorem 2 .

\section{References}

[1] J. Bourgain, Global Solutions of Nonlinear Schrödinger Equations, Amer. Math. Soc., Providence, 1999.

[2] Y. Brenier, Converence of the Vlasov-Poisson system to the incompressible Euler equations, Commun. Partial Diff. Eqs., 25 (2000), 737-754.

[3] F. Brezzi and P. Markowich, The three dimensional Wigner-Poisson problem: existence, uniqueness and approximation, Math. Methods Appl. Sci., 14 (1991), 35-62.

[4] L. C. Evans, Weak Convergence Methods for Nonlinear Partial Differential Equations, Amer. Math. Soc., Providence, 1990.

[5] I. Gasser, L. Hsiao, P. Markowich and S. Wang, Quasineutral limit of a nonlinear drift-diffusion model for semiconductor models, To appear in J. Math. Anal. Appl., 2002.

[6] I. Gasser, C. K. Lin and P. Markowich, A review of dispersive limits of Nonlinear Schrödinger-type equations, Taiwanese J. of Math., 4 (2000), 501-529.

[7] I. Gasser, C. D. Levermore, P. Markowich and C. Schmeiser, The initial time layer problem and the quasineutral limit in the semiconductor drift-diffusion model, Europ. J. Appl. Math., 12 (2001), 497-512.

[8] I. Gasser and P. Markowich, Quantum hydrodynamics, Wigner transforms and the classical limit, Asympt. Anal., 14 (1997), 97-116.

[9] P. Gérard, P. Markowich, N. Mauser and F. Poupaud, Homogenization limits and Wigner transforms, Commun. Pure Appl. Math., 50 (1997), 323-379. 
[10] E. Grenier, Oscillations in quasineutral plasmas, Commun. Partial Diff. Eqs., 21 (1996), 363-394.

[11] E. Grenier, Defect measures of the Vlasov-Poisson system, Commun. Partial Diff. Eqs., 20 (1995), 1189-1215.

[12] E. Grenier, Pseudodifferential estimates of singular perturbations, Commun. Pure Appl. Math., 50 (1997), 821-865.

[13] N. Hayashi and H. Hirata, Global existence of small solutions to nonlinear Schrödinger equations, Nonlin. Anal. TMA, 31 (1998), 671-685.

[14] S. Jin, C. D. Levermore and D. W. Mclaughlin, The semiclassical limit of the defocussing NLS hierarchy, Commun. Pure Appl. Math., 52 (1999), 613-654.

[15] A. Jüngel and Y.-J. Peng, A hierarchy of hydrodynamic models for plasmas: quasineutral limits in the drift-diffusion equations, Asympt. Anal., 28 (2001), 49-73.

[16] L. D. Landau and E. M. Lifschitz, Lehrbuch der Theoretischen Physik, Vol. III (Quantenmechanik), Akademie-Verlag, Berlin, 1985.

[17] M. L. Loffredo and L. M. Morato, Self-consistent hydrodynamical model for HeII near absolute zero in the frame work of stochastic mechanics, Phys. Rev. B, 35 (1987), 1742-1747.

[18] P. L. Lions and T. Paul, Sur les mésures de Wigner, Revista Mat. Iberoamericana, 9 (1993), 553-618.

[19] P. L. Lions, B. Perthame and E. Tadmor, Kinetic formulation for the isentropic gas dynamics and p-system. Commun. Math. Phys, 163 (1994), 415-431.

[20] F. H. Lin and P. Zhang, On the hydrodynamic limit of wave Ginzburg-Landau vortices, Preprint, Beijing, China, 2001.

[21] A. Majda, Compressible Fluid Flow and Systems of Conservation laws in Several Space Variables, Springer-Verlag, Berlin, 1984.

[22] P. Markowich and N. Mauser, The classical limit of a self-consistent quantum-Vlasov equation in 3D, Math. Models Methods Appl. Sci., 3 (1993), 109-124.

[23] P. Markowich, C. Ringhofer and C. Schmeiser, Semiconductor Equations, SpringerVerlag, Vienna, 1990.

[24] M. Puel, Etudes Variationnelles et Asymptotiques des Problèmes de la Mécanique des Fluides et des Plasmas, PhD thesis, Université Paris 6, 2000.

[25] W. A. Strauss, The Nonlinear Wave Equation, Amer. Math. Soc., Providence, 1989. 
[26] L. Tartar, H-measures, a new approach for studing homogenization, oscillations and concentration effects in partial differential equations, Proc. Roy. Soc. Edinb., 115A (1990), 193-230.

[27] M. Taylor, Pseudodifferential Operators, Princeton University Press, Princeton, 1981.

[28] Z. P. Xin and P. Zhang, On the global existence of weak solutions to the shallow water equation, Commun. Pure Appl. Math., 53 (2000), 1411-1433.

[29] P. Zhang, Wigner measure and the semiclassical limit of Schrödinger-Poisson equations, Preprint, 2001.

[30] P. Zhang, Y. X. Zheng and N. Mauser, Semiclassical limit of Schrödinger-Poisson to Vlasov-Poisson equations with general data in one dimension, To appear in Commun. Pure Math. Appl., 2002. 